(C) 2016 IEEE. Personal use of this material is permitted. Permission from IEEE must be obtained for all other uses, in any current or future media, including reprinting/republishing this material for advertising or promotional purposes, creating new collective works, for resale or redistribution to servers or lists, or reuse of any copyrighted component of this work in other works. 
$1 \quad$ An Inflatable and Wearable Wireless System for Making 32-Channel

\section{Electroencephalogram Measurements}

3

4 Yi-Hsin $\mathrm{Yu}^{1,2}$, Shao-Wei $\mathrm{Lu}^{2}$, Chun-Hsiang Chuang ${ }^{2}$, Jung-Tai King ${ }^{2}$, Che-Lun Chang ${ }^{2}$, 5 Shi-An Chen ${ }^{2}$, Sheng-Fu Chen ${ }^{2}$ and Chin-Teng Lin ${ }^{1,2,3^{*}}$, Fellow, IEEE

6

$7{ }^{1}$ Institute of Electrical Control Engineering, National Chiao Tung University, Hsinchu 300, 8 Taiwan.

$9 \quad{ }^{2}$ Brain Research Center, National Chiao Tung University, Hsinchu 300, Taiwan.

$10{ }^{3}$ Faculty of Engineering and Information Technology, University of Technology, Sydney, 11 Australia.

Text pages: 32 ; Number of figures and tables: 8

*Correspondence should be addressed of the following:

Chin-Teng Lin, Ph.D.,

Institute of Electrical Control Engineering,

19 Tel: +886-3-571-2121 ext 31753; E-mail: ctlin@ mail.nctu.edu.tw 


\section{Abstract}

2

3

Potable electroencephalography (EEG) devices have become critical for important research. They have various applications, such as in brain computer interfaces (BCI). Numerous recent investigations have focused on the development of dry sensors, but few concern the simultaneous attachment of high-density dry sensors to different regions of the scalp to receive qualified EEG signals from hairy sites. An inflatable and wearable wireless 32-channel EEG device was designed, prototyped, and experimentally validated for making EEG signal measurements; it incorporates spring-loaded dry sensors and a novel gasbag design to solve the problem of interference by hair. The cap is ventilated and incorporates a circuit board and battery with a high-tolerance wireless (Bluetooth) protocol and low power consumption characteristics. The proposed system provides a 500/250 Hz sampling rate, and 24 bit EEG data to meet the BCI system data requirement. Experimental results prove that the proposed EEG system is effective in measuring audio event-related potential (AERP), measuring visual event-related potential (VERP), and rapid serial visual presentation (RSVP). Results of this work demonstrate that the proposed EEG cap system performs well in making EEG measurements and is feasible for practical applications.

8 Keywords: electroencephalography (EEG), brain-computer interface, audio event-related 9 potential (AERP), visual event-related potential (VERP), rapid serial visual presentation $20 \quad(\mathrm{RSVP})$ 
Electroencephalography (EEG) has been extensively utilized in neuroscience research

3 4 diagnostics and neurobiological research [2, 3], because EEG is non-invasive, has a high

5

6

7 and rehabilitation engineering [1]. The traditional EEG system was developed for medical temporal resolution and is portable [4-6]. As technology advances, better EEG systems are developed. EEG measurement has become one of most convenient and practical tools for measuring brain dynamics. With the development of cognitive brain science, many tools for interpreting images of the brains and signals have been sought. Additionally, the EEG-based brain-computer interface $(\mathrm{BCI})$ system has become more popular [7-9]. EEG is no longer required only on a single point or one part of the brain. Multiple-channel EEG measurements are increasingly required.

Traditional EEG systems are not very convenient. For examples, many products for use in the laboratory, such as Neuroscan (Compumedics USA Inc., Charlotte, NC), ActiveTwo (BioSemi Inc., Amsterdam, Netherlands) and Brain Products' EEG devices (Brain Products GmbH Inc., Gilching, Germany) required experts to operate because the installation, calibration and assessment of the system before it can be used to measure EEG signals require expert knowhow. These products are powerful in professional EEG measurement but may not as electronic consumer products. 
In addition, traditional EEG systems are inconvenient for use in everyday life owing to

2 their bulkiness and wired data acquisition. During the EEG measurement procedure, users

3 must complete a procedure in a room, preventing them from going to the toilet freely. Some

4 medical EEG products use wet EEG sensors to yield highly accurate brain dynamics because

5 wet EEG sensors overcome interference by hairs and provide qualified EEG signals, but

6 these products are is not convenient for users, because subjects must clean their hair and

7 scalp after the EEG measurements are made.

8 Therefore, demand for a portable and mobile EEG system is rising. EEG systems for

9 everyday use have recently become popular. Many EEG system manufacturers make wireless

10 EEG systems, which allow users can to move freely while carrying portable devices (with an

11 EEG receiving application). Moreover, an increasing number of EEG system manufacturers

are announcing the production of dry EEG sensors to eliminate the inconvenience of cleaning

the hair and scalp after use. Several studies have focused on the development of dry sensors,

14 but only a few have addressed the simultaneous attachment of high-density dry sensors to various regions of the scalp to receive qualified EEG signals from hairy sites. The three general methods of attaching dry EEG electrodes are as follows.

1) Using tapes, ties or a headband to attach electrodes to the scalp. This method can be 
1 Nevertheless, it attaching high-density EEG electrodes to the scalp, as for 32- or 64-channel

2 EEG measurement, is difficult.

2) Using elastic branches to attach electrodes to the scalp. As an example, NeuroFocus (USA Inc., Berkeley, CA) released the first wireless full-brain EEG headset called "Mynd" [11] which is more fast and convenient application. Although this method cannot provide precision pressure control in each nodes, which would allow for multiple degrees of freedom to conform to the variations of head shapes and sizes. In addition, branches may suffer elastic fatigue problem after long-time use.

3) Using fully sheathed elastic headgear to attach electrodes to the scalp. For example, the g.GAMMA cap (Cortech Solutions, USA Inc., Wilmington, NC) with highly optimized fit to the head and very narrow joints to maximize possible electrode locations. In this way, EEG cap provides the properly or ideally effect for electrodes attachment. This mechanism is also widely used in different EEG cap products. But the disadvantages are the senses of tightness, air impermeability and uncomforting for the scalp.

This study proposed a 32-channels inflatable and wearable wireless EEG system. The EEG cap with a mechanism of spring-loaded dry electrodes and an inflatable gasbag is convenient, comfortable and quick to put on. It has four major advantages. First, the pogo-probes of spring-loaded dry sensors overcome hair interference and contact effectively the skin of the scalp. These dry sensors can be firmly attached to various parts of the scalp 
1 and can receive qualified EEG signals even at hairy sites. The dry sensors are resilient and

2 can be used repetitively on hairy sites without conductive gel. In particular, the pogo-probes

3 on the electrodes can retract fully into their housing, making the design safer. Second, the

4 gasbag that is located between the shell of the EEG cap and the elastic branches improves the

5 contact between all sensors to and parts of the scalp with various shapes. Inflating the gasbag

6 forces the branches and sensors down to touch the scalp, regardless of the shape and size of

7 the head. Third, EEG data can be wirelessly received by portable devices, such as laptops,

8 smartphones, or tablets, through the Bluetooth protocol without external devices or cables.

9 Fourth, the EEG acquisition module is small and light. The whole EEG circuit consumes

10 relatively little power, and is embedded into the EEG cap without exposed wires.

11 Experimental results demonstrate that the proposed EEG system performs well in EEG

12 acquisition and is feasible for BCI applications. 


\section{A. Dry Sensors}

Two dry sensors are used for forehead and hairy sites. Spring-loaded sensors [12] with

4 an array of probes of different lengths are used at hairy sites. The probes on the electrodes can retract fully into their housing, making the design safe, overcoming the obstruction by hair and providing adequate scalp contact that does not hurt the skin. Dry spring-loaded EEG sensors, shown in Fig. 1(A), with 16 pogo-probes, are used. The length of the spring-loaded can be adjusted to provide effective scalp contact at hairy sites with various densities and lengths of hair. In general, the shorter probes are applied to the temporal lobe, and the longer probes are applied to the parietal lobe. The top of each probe is spheroid to avoid stinging the skin and it is coated with gold with low impedance (approximately $30 \mathrm{~m} \Omega$ ). The substrate and pad of the sensor provide a buffer effect, preventing pain when force is applied. The spring force of the sensor is approximately $20 \mathrm{~g}$. The effects of the spring-loaded sensor were verified and presented in $[12,13]$. The other form of dry sensor is the foam-based sensor, which is used on the forehead, as presented in Fig. 1(B) [14]. It has flexible material that supported comfortably tactile impression with low contact impedance. Both of the developed sensors can be used to measure bio-potentials without special preparation of the skin $[15,16]$ or the use of conduction gel. The ground and reference electrodes were designed as clips, as 
1 shown in Fig 3(E), and located at the earlobe. These two sensors are more convenient for

2 making EEG measurements than are conventional wet electrodes.

4 B. Circuit Design

5

The typical potential of EEG signal is approximately 10-6 V. This potential is very small

6 so must be amplified before analysis. Since artifacts can easily affect EEG signal

7 measurement, the amplification of the brain potential will also amplify the noise signals [17].

8 Two-level amplifiers and some filters designs are included in the circuit to reduce

9 interference by artifacts and improve the quality of EEG measurements that are made using a

10 dry sensor. Figure 1 (C) shows the circuit device. The major components include 1)

11 instrumentation amplifiers, 2) analog-to-digital converter (ADC) units, 3) a microcontroller

12 unit and 4) a wireless module (Bluetooth unit). The size of the proposed circuit board was 65

$13 \times 35 \times 4 \mathrm{~mm}^{3}$. The circuit board and battery were small enough to be embedded into

14 proposed EEG cap. In the EEG measurement process, analog EEG signals were measured

15 using the dry sensors and pre-amplified by instrumentation amplifiers. The preamplifier

16 provided high input impedance and a high common-mode rejection ratio (CMRR) and

amplified the microvolt-level EEG signals to a detectable range. Then, the analog EEG signal 
1 digital data of each channel into Bluetooth packets. The packets were switched to a

2 Bluetooth module via a universal asynchronous receiver/transmitter (UART).

The gain of the preamplifier unit was set to 1361(default), and the cut-off frequency was regulated to $0.23 \mathrm{~Hz}$ by a high-pass filter. The ADC setting was configured to provide 24 bit resolution and 500/250 Hz sampling rate translation. The power-line interference could be removed by a micro-controller using a moving average filter (notch filer) with a frequency of $60 \mathrm{~Hz}$. The digitalized and processed signals are transmitted to receiving application via a Bluetooth module with a baud-rate of 921600 bits/s. Power was supplied by a high capacity (750 mAh, 3.0 V) Li-ion battery that provided $8 \sim 10$ hours continuous operation. Figure 2(A) shows the design of EEG signal acquisition and processing flow diagram. Figure 2(B) shows the specifications of the proposed EEG system with their performance values.

\section{EEG Cap Mechanism Design}

The proposed EEG cap design consists of three parts - horizontal, vertical, left and right parts. Crawler ring design is in horizontal position with spring-loaded sensors is shown in Fig. 3(A). The sensors placement follows the international 10-20 system [19, 20]. EEG cap has a tightness adjusting knob mechanism in the backend as shown in Fig. 3(B). By tightening the ring, dry sensors can be made to fit the scalp closely. The outside view of the crawler design is shown in Fig 3(C), which is based on the human spine. The crawler design accommodates 
1 different head shapes to ensure close contact with the scalp. Sensors are located in the

2 crawler arc according to the 10-20 system and results for locating electrodes. The EEG cap

3 also has a knob for adjusting the vertical length of the arc. The inside curved elastic ring

4 design with spring-loaded sensors is shown in Fig. 3(D). The middle of the C-type elastic

5 ring is connected to the central crawler. This design has a fishbone pattern. When the central

6 arc headband is tightened to bring it into close contact with the scalp, the end points of the

7 C-ring are forced down to touch the scalp. This design ensures effective contact between the

8 sensors and the scalp. The ground and reference electrodes, shown in Fig. 3(E), are designed

9 as clips for the earlobe. A chin strap is used to secure the EEG cap, as shown in Fig. 3(F),

10 preventing the EEG cap from rising when the gasbag is inflated. This design closely aligns

11 the fishbone mechanism with the scalp upon inflation.

Elastic branches are used to attach electrodes to the scalp; these may suffer from elastic

fatigue after long-time use. This work develops the gasbag design to solve this problem and to ensure that the EEG cap is effective for differently shaped scalps. The prototype gasbag is based on a beach ball. After several tests and improvements, the design was workable and improved the operation of the elastic branches. The gasbag is positioned between the shell of the cap and the branches, to which the spring-loaded dry electrodes are attached. When the gasbag inflates, it forces the branches and sensors downward to touch the scalp. The probes of the spring-loaded dry sensors overcome hair interference to touch safely the skin on the 
1 scalp. Figure 4(A) shows the final gasbag configuration. The gasbag is attached to the crevice

2 between the periphery of the cap and the central fishbone. The gasbag can expand by

3 approximately $2-3 \mathrm{~cm}$, as shown in Fig. 4(B). Figure 4(C) shows that before the gasbag is

4 inflated, some sensors do no touch the scalp. Figure 4(D) shows that when the gasbag is

5 inflated, it can press the fishbone mechanism more firmly into the scalp, improving the

6 contact between the sensors and the scalp. This design also overcomes the problem of

7 impermeability to air.

9 D. Firmware and Receiving Application

The skin-electrode interface can generate an additional DC offset of approximately 300

$\mathrm{mV}$ [21]. This offset can be eliminated to prevent saturation of the amplifier. In this system,

12 the bandwidth of the device was between $0.23 \mathrm{~Hz}$ and $125 \mathrm{~Hz}$. The ADC provided data

resolution of 24bit /channel. A 16 bit microcontroller was utilized. Special attention was paid

to the processing of $24 \mathrm{bit} / \mathrm{channel}$ data on a 16 bit microcontroller. Also, a high pass filter

with a $125 \mathrm{~Hz}$ cutoff frequency was used. The ADC provided a maximum sampling rate of

$500 \mathrm{~Hz}$, yielding a conversion time of $2 \mathrm{~ms}$, during which interval the EEG signal could be

acquired and conditioned. The internal data translation was triggered by a microcontroller interrupter. The signal from the ADC was transmitted to an interrupt pin in the microcontroller. When an interrupt signal was received, the digitized EEG signal was 
1 acquired via the serial port interface (SPI) interface. Then, the microcontroller packed the

2 EEG data as a formatted Bluetooth packet. The packet schema included a header, a command,

3 the resolution and sampling rate, the gain and channels, and an end item. The end item of the

4 packet also contained bit-check and some control signal information.

In the EEG signal processing, EEG signals were firstly received by dry sensors. Then,

6 the proposed circuit amplified the signals, converted them from analog to digital and then

7 filtered them. Finally, the raw EEG data were transmitted through a wireless module

8 (Bluetooth protocol) and received by a smartphone, notebook or tablet computer. Users can

9 easily wear the EEG cap without skin abrasion or preparation, and the skin does not have to

10 be cleaned after it is worn. The battery power supply and the wireless design provide more

11 comfort for the user, eliminating interference by cables. The main results of this research can

12 be utilized for remote/home care, strength training games, clinical applications, BCI, and experiments in cognitive neuroscience [14, 22-24]. 
1 Results

2 A. Basic Electrical Test of the Sensor and Circuit

EEG signals range from 10 to $100 \mu \mathrm{v}$. A validation test was used to confirm the quality

4 of the signals. Input data were fed into a programmable function generator and passed

5 through a voltage divider, yielding simulated human EEG signals. Two tests was performed

6 with input signals of 50 and $100 \mu \mathrm{v}$ (VPP) and frequencies of $2 \mathrm{~Hz}, 5 \mathrm{~Hz}, 20 \mathrm{~Hz}$ and $80 \mathrm{~Hz}$. In

7 the EEG acquisition application, the sampling rate was set to $250 \mathrm{~Hz}$ and the gain was 1 .

8 After several seconds of measurement, $1 \mathrm{~s}$ of results were fetched in Fig. 5 (A). The

9 differences between the maximum and minimum amplitudes are approximately $50 \mu \mathrm{v}$ (blue

10 curve) and $100 \mu \mathrm{v}$ (green curve). The results were as expected. Figure 5(B) shows the spectral

11 scan of the spring-loaded sensor. The frequencies of the input signals were from $20 \mathrm{~Hz}$ to

$121000 \mathrm{~Hz}$. Since the spring-load sensor has coils, inductance is always present, causing the

13 impedance to increase with frequency. Comparing the impedances of the spring-loaded

14 sensor and the preamplifier reveals that the spring-loaded sensor impedance is small enough

15 to be ignored. The experimental results indicate that the spring-loaded sensor performed well and stably.

\section{B. Basic EEG Signal Measurement}

When the proposed EEG cap is worn, the gasbag cannot be inflated. Therefore, the

20 quality of the signals in some channels was poor. Information about poor signals was 
1 obtained manually. The left part of Figs. 6(A) and 6(B) show poor signals from two subjects.

2 The noisy pattern of each channel shows that the sensor was not in good contact with the

3 scalp. Based on experienced in the laboratory, poor channel signals were usually received in

4 the vertical (center bay) or the left and right sides of the cap. The channels of horizontal ring

5 worked well because there was a knob mechanism enabled the size of the horizontal ring to

6 be adjusted to force the sensors into contact with the scalp. In contrast, sensors on the vertical

7 side, and both left and right sides functioned poorly owing to variation in the shape of the

8 scalp. The original fishbone mechanism did not provide good contact between the sensors

9 and the scalp. In this study, the gasbag mechanism was adopted to solve the problem. When

10 the gasbag was inflated, it pressed the sensors down into contact with the scalp, as shown in

11 Fig. 4(D). The jaw artifacts patterns are presented in the right part of Figs. 6(A) and 6(B). It

12 illustrates that the proposed EEG cap achieve good contact between the dry sensors and the 13 scalp.

\section{EEG Signal Comparisons}

A typical alpha rhythm testing $[8,14,25]$ was performed. For the $(\mathrm{Oz})$ position alpha rhythm measurements, Fig. 7(A) shows during resting state, the EEG signals of the eyes 
1 comparison of the alpha power $(8 \sim 12 \mathrm{~Hz})$ between eyes opening (red curves) and eyes

2 closing (blue curves) cases. The alpha phenomena were observed in this EEG system.

4 D. EEG Oddball Experiment for Signal Quality verification

5 Oddball tasks are common EEG experiments which are used to verify whether the EEG

6 signal quality is sufficient $[7,26]$. Two experiments (audio oddball and visual oddball

7 experiments) were conducted, and their EEG signals were recorded with the proposed EEG

8 system. The event-related potential (ERP) maps were evaluated using EEGLAB (v10.2.5.5b)

9 and MATLAB [27].

10

The P200 component was a positive detection with typical peak latency of approximately $150-250 \mathrm{~ms}$ elicited by auditory stimuli $[28,29]$. For the (T8) position EEG signal measurements, approximately 250 trials were performed, and clear P200 components

13 (i.e., the peaks indicated as p200) were detected while acquiring the ERP map shown in Fig 8(A).

The P300 ERP occurred during visual stimuli during two different tasks. The oddball paradigm presented both target and standard stimuli [30]. For the (O1) position EEG signal measurements, the trials number was about 250, and clear P300 components (with peaks indicated as p300) were detected while acquiring the ERP map shown in Fig 8(B). 


\section{E. The RSVP Experiment for EEG Signal Quality Verification}

This section demonstrates the proposed system in a typical P300 task using a rapid serial visual presentation (RSVP) image display paradigm, which is an experimental model that is frequently used to examine the temporal characteristics of attention in various BCI applications, such as deception detection [31]. The RSVP is a fast-content recognition approach that uses EEG to record brain activity that is elicited by fast bursts of image data [32]. In Fig. 9(A), the frequency of every stimulus is $5 \mathrm{~Hz}$, so the duration of every presented character is $200 \mathrm{~ms}$. The letter "G" is the target. The number of trails of whole experiment is 80. Eight subjects participated in this task to evaluate the functions of the developed system. ERP maps were evaluated using EEGLAB (v10.2.5.5b) and MATLAB also. All of their experimental RSVP results were conspicuous. For example, Fig. 9(B) displays the clear RSVP ERP map of one subject. To verify that all channels of the proposed EEG system could function simultaneously, the experimental data were used to perform an ICA analysis. Three pieces of extremely poor channel information were removed in this process. Figure 9(C) shows the potential of 29 channels, and Fig. 9(D) shows the ICA dipole map. Results of these EEG signals were recorded and analyzed by proposed EEG system. 


\section{Conclusions}

The proposed EEG system is a novel, inflatable, dry, and 32-channel wireless EEG cap system that can be applied to the scalp without any skin preparation or the use of conductive gel. This system was studied, designed and tested. The advantages of this EEG system are summarized as follows. First, the EEG cap is ergonomic and equipped with dry sensors that can be used without gel. Second, the system generates high-density and high-quality EEG signals that can be used for research into brain dynamics. The experimental results herein confirm that the proposed EEG system can be used to measure the EEG signals of the human brain. Generally, the setup time of the proposed EEG system is less than 20 minutes. The power was supplied by a high-capacity $(750 \mathrm{mAh}, 3.0 \mathrm{~V}) \mathrm{Li}$-ion battery that provided (8 10 OR eight to ten) hours of continuous operation.

Although the proposed EEG system has many advantages, room exists for improvement, because major dry sensors are made of metal pogo-probes that may cause discomfort to users after several hours of application. Non-mental dry sensors that can be used on hairy sites should be developed.

\section{Acknowledgements}

This work was supported in part by the UST-UCSD International Center of Excellence in Advanced Bio-engineering sponsored by the Taiwan National Science Council I-RiCE 
1 Program under Grant Number: NSC-102-2911-I-009-101; in part by the Aiming for the Top

2 University Plan of National Chiao Tung University, the Ministry of Education, Taiwan; and

3 in part by the Army Research Laboratory and was accomplished under Cooperative

4 Agreement Number W911NF-10-2-0022. The views and the conclusions in this document

5 are those of the authors and should not be interpreted as representing the official policies,

6 either expressed or implied, of the Army Research Laboratory or the U.S. Government. The

7 U.S. Government is authorized to reproduce and distribute reprints for Government purposes

8 notwithstanding any copyright notation herein. 
2 Figure 1. (A) The proposed dry EEG spring-loaded sensor with size specification. (B) The

3 dry foam-based EEG sensor. (C) Schematic circuit board diagrams of Instrumentation

4 amplifier, Bluetooth model, ADC and Microcontroller for implementation of wireless EEG

5 acquisition.

6

7 Figure 2. (A) Design of EEG signal acquisition and processing flow diagram. The real-time

8 EEG signal monitoring application is implemented by portable devices such as smartphone,

9 notebook or tablet computer without external devices. (B) The specifications of the proposed

10 EEG system with their performance values.

12 Figure 3. The mechanism and components of the proposed EEG cap. (A) Crawler ring

13 design in horizontal position with spring-loaded sensors. (B) The tightness adjusting knob

14 mechanism. (C) The outside view of the crawler design. (D) The inside curved elastic ring design with spring-loaded sensors. (E) The ground and reference electrodes for both ears. (F)

16 A chin strap design.

18 Figure 4. (A) The EEG cap prototype appearance and its embedded gasbag. (B) The inside view of the EEG cap. There is about $4 \mathrm{~cm}$ space between shell and elastic branches when the gasbag is inflated. (C) Pink area is the gasbag. Before the gasbag is inflated, some of the 
1 sensors do not have a good contact with the scalp. (D) After the gasbag is inflated, the gasbag

2 would force the branches downward such that sensors would have good contact with the

3 scalp.

5 Figure 5. (A) Basic electrical test results of the proposed circuit. Two VPP input signals of

$650 \mu \mathrm{v}$ and $100 \mu \mathrm{v}$, and different frequency input signals of $2 \mathrm{~Hz}, 5 \mathrm{~Hz}, 20 \mathrm{~Hz}$ and $80 \mathrm{~Hz}$ were

$7 \quad$ applied. (B) The spectrum scanning result of the spring-loaded sensor.

9 Figure 6. The left part of (A) indicates subject 1 yields 4 low quality channels signals (F4, F3,

$10 \mathrm{CZ}$, and CPZ) due to insufficient contact between sensors and the scalp. However, right part

11 of (A) indicates those sensors were workable after the gasbag was inflated. The jaw artifacts patterns are presented. Similarly, (B) shows subject 2 has same effects as the subject 1 in (A).

Figure 7. (A) During resting state, the EEG signals of the eyes opening (red curve) and closing (blue curve) within $10 \mathrm{~s}$ window. (B) Alpha wave re-placement after processing the FFT transformation.

Figure 8. EEG raw data were preprocessed by $50 \mathrm{~Hz}$ high-pass filter, $1 \mathrm{~Hz}$ low-pass filter and 
1 T8. The P200 phenomena are presented in this diagram. (B) Shows a visual oddball ERP

2 mapped with a dry sensor located at $\mathrm{O} 1$. The P300 phenomena are presented in this diagram.

4 Figure 9. (A) The RSVP experiment design diagram. Only one of the alphabetic letters can

5 be presented in a fixed location of screen. When the letter " $G$ " is presented, subject must

6 press the count button. The inter-burst interval is $0.2 \mathrm{~s}$. After the sequence is presented, the

7 user is asked to indicate the number count of letter "G". (B) One of the subject's (subject 1)

8 ERP result of RSVP. In this analysis, PZ channel's EEG data was used. The clear P300 wave

9 is presented. (C) Using the same experimental data of subject 1, 29 channels' EEG data were

10 reserved after the removal of bad channels EEG signals. The EEG potential/ time analysis

11 result is shown. (D) After ICA analysis, the dipole maps are presented. 


\section{References}

[1] N. V. Thakor, Biopotentials and Electro-Physiology Measurement. In The Measurement, Instrumentation, and Sensors Handbook. FL,USA: CRC Press: Boca Raton, 1999.

[2] C. Miniussi and G. Thut, "Combining TMS and EEG offers new prospects in cognitive neuroscience," Brain Topography, vol. 22, pp. 249-256, Jan 2010.

[3] N. Srinivasan, "Cognitive neuroscience of creativity: EEG based approaches," Methods, vol. 42, pp. 109-116, May 2007.

[4] M. B. Shulman, "The clinical applications of EEG: origins," Epilepsy \& Behavior, vol. 3, pp. 393-394, Aug 2002.

[5] S. Machado, et al., "EEG-based brain-computer interfaces: an overview of basic concepts and clinical applications in neurorehabilitation," Reviews in the Neurosciences, vol. 21, pp. 451-468, 2010.

[6] Y. C. Chen, et al., "Spatial and temporal EEG dynamics of motion sickness," Neuroimage, vol. 49, pp. 2862-2870, Feb 2010.

[7] T. O. Zander, et al., "A dry EEG-system for scientific research and brain-computer interfaces," Frontiers in Neuroscience, vol. 5, pp. 1-10, May 2011.

[8] T.-H. Huang, et al., "Development of a Brain-Controlled Rehabilitation System (BCRS)," Journal of Neuroscience and Neuroengineering, vol. 2, pp. 79-89, Apr 2013.

[9] S. G. Mason, et al., "A comprehensive survey of brain interface technology designs," Annals of Biomedical Engineering, vol. 35, pp. 137-169, Feb 2007.

[10] Y. M. Chi, et al., "Dry and noncontact EEG sensors for mobile brain-computer interfaces," IEEE Transactions on Neural Systems and Rehabilitation Engineering, vol. 20, pp. 228-235, Mar 2012.

[11] PRNewswire. (2011). NeuroFocus Announces World's First Wireless Full-Brain EEG Measurement Headset: Mynd ${ }^{\mathrm{TM}}$.

Available: http://www.prnewswire.com/news-releases/neurofocus-announces-worlds-first-wireless-full-brain-eegmeasurement-headset--mynd-118355014.html

[12] L.-D. Liao, et al., "Design, fabrication and experimental validation of a novel dry-contact sensor for measuring electroencephalography signals without skin preparation," Sensors, vol. 11, pp. 5819-5834, Jun 2011.

[13] L.-D. Liao, et al., "A novel 16-channel wireless system for electroencephalography measurements with dry spring-loaded sensors," IEEE Transactions on Instrumentation and Measurement, vol. 63, pp. 1545-1555, Jun 2014.

[14] L.-D. Liao, et al., "Gaming control using a wearable and wireless EEG-based brain-computer interface device with novel dry foam-based sensors," Journal of NeuroEngineering and Rehabilitation, vol. 9, pp. 1-12, Jan 2012.

[15] S. Farshchi, et al., "A TinyOS-enabled MICA2-based wireless neural interface," IEEE Transactions on Biomedical Engineering, vol. 53, pp. 1416-1424, Jul 2006. 
[16] Y. S. Kim, et al., "Helmet-based physiological signal monitoring system," European Journal of Applied Physiology, vol. 105, pp. 365-372, Feb 2009.

[17] W. O. Tatum, et al., "Artifact and recording concepts in EEG," Journal of clinical neurophysiology, vol. 28, pp. 252-263, Jun 2011.

[18] D. Campillo, et al., "A real time ECG preprocessing system based on ADS1298," in Computing in

[19] K. Gramann, et al., "Cognition in action: imaging brain/body dynamics in mobile humans," Reviews in

[20] U. Herwig, et al., "Using the international 10-20 EEG system for positioning of transcranial magnetic

[21] M. B. Karthik Soundarapandian, "Analog front-end design for ECG systems using Delta-Sigma ADCs," Texas Instruments, Application Report, Apr 2010.

[22] R. N. Khushaba, et al., "Consumer neuroscience: Assessing the brain response to marketing stimuli using electroencephalogram (EEG) and eye tracking," Expert Systems with Applications, vol. 40, pp. 3803-3812, Jul 2013.

[23] C.-T. Lin, et al., "Review of wireless and wearable electroencephalogram systems and brain-computer interfaces--a mini-review," Gerontology, vol. 56, pp. 112-119, Jul 2010.

[24] L.-D. Liao, et al., "Biosensor technologies for augmented brain-computer interfaces in the next decades," Proceedings of the IEEE, vol. 100, pp. 1553-1566, May 2012.

[25] B. J. Lance, et al., "Brain-computer interface technologies in the coming decades," Proceedings of the IEEE, vol. 100, pp. 1585-1599, May 2012.

[26] Y.-H. Yu, et al., "Design, fabrication, and experimental validation of novel flexible silicon-based dry sensors for electroencephalography signal measurements," IEEE Journal of Translational Engineering in Health and Medicine, vol. 2, pp. 1-7, Nov 2014.

[27] A. Delorme and S. Makeig, "EEGLAB: an open source toolbox for analysis of single-trial EEG dynamics including independent component analysis," Journal of Neuroscience Methods, vol. 134, pp. 9-21, Mar 2004.

[28] B. A. Brett-Green, et al., "Multisensory integration in children: a preliminary ERP study," Brain Research, vol. 1242, pp. 283-290, Nov 2008.

[29] F. Ferreira-Santos, et al., "The auditory P200 is both increased and reduced in schizophrenia? A meta-analytic dissociation of the effect for standard and target stimuli in the oddball task," Clinical Neurophysiology, vol. 123, pp. 1300-1308, Jul 2012.

[30] J. Polich and C. Margala, "P300 and probability: comparison of oddball and single-stimulus paradigms," International Journal of Psychophysiology, vol. 25, pp. 169-176, Feb 1997.

[31] C. Hope, et al., "High throughput screening for mammography using a human-computer interface with rapid serial visual presentation (RSVP)," in Medical Imaging 2013: Image Perception, Observer Performance, and Technology Assessment, Lake Buena Vista (Orlando Area), Florida, USA, 2013, pp. 867303-867308. 
H. Bowman, et al., "Subliminal salience search illustrated: EEG identity and deception detection on the fringe of awareness," PLoS ONE, vol. 8, pp. 1-21, Jan 2013.

3

4 
$1 \quad$ Figure

$2 \quad$ Figure 1.

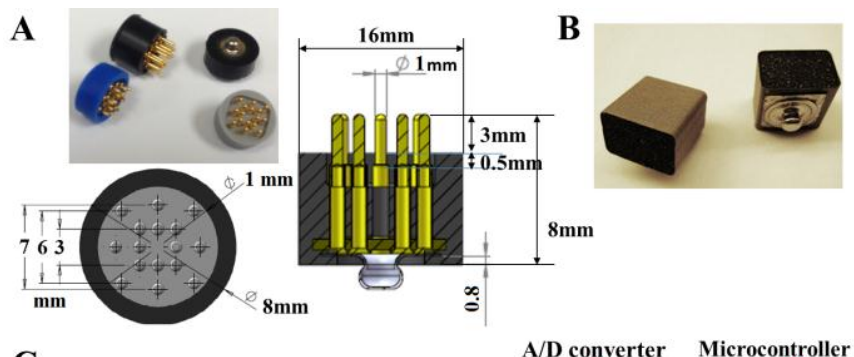

C Instrumentation amplifier Bluetooth model

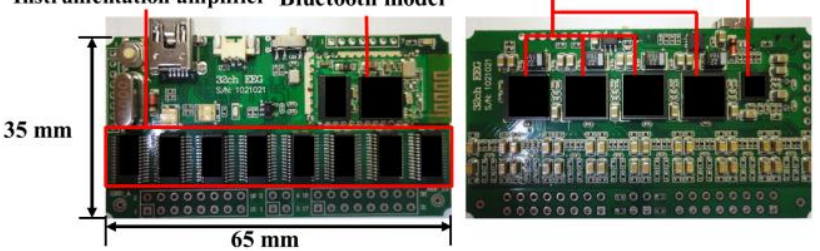

3 
$1 \quad$ Figure 2.

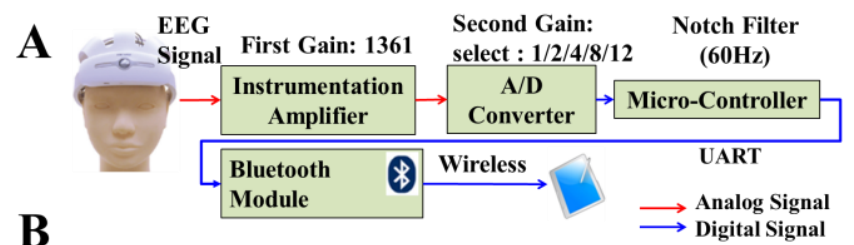

\begin{tabular}{|c|c|}
\hline Specifications & Values \\
\hline Sampling Rate & $500 / 250 \mathrm{~Hz}$ \\
\hline Resolution & $16 / 24$ bits \\
\hline Bandwidth & $0.23 \sim 125 \mathrm{~Hz}$ \\
\hline $\begin{array}{l}\text { Instrumentation } \\
\text { Amplification Factor }\end{array}$ & 1361 \\
\hline $\begin{array}{l}\text { Second Amplification } \\
\text { Factor }\end{array}$ & $1 / 2 / 4 / 8 / 12$ \\
\hline Battery & $3.7 \mathrm{~V}, 750 \mathrm{MA}$ \\
\hline Battery Life & 8 10 hours \\
\hline CMMR & $110 \mathrm{~dB}$ \\
\hline Event Marker Latency & $50 \mathrm{~ms}$ \\
\hline Output Current & $62 \mathrm{~mA}$ \\
\hline Output File Type & edf/ bdf/ txt/ cnt/ csv \\
\hline $\begin{array}{l}\text { Hardware } \\
\text { Compatibility }\end{array}$ & Desktop/Laptop/Android Pad/Android Phone \\
\hline OS Compatibility & Up to Windows 8 / Android 2.0 or higher \\
\hline
\end{tabular}


$1 \quad$ Figure 3.

2

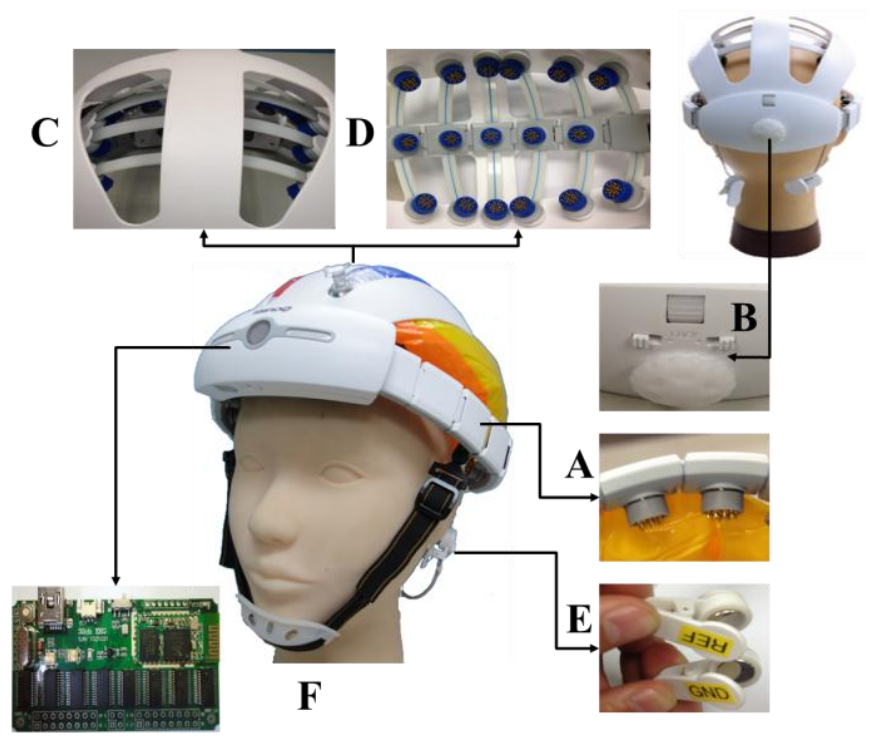


$1 \quad$ Figure 4.

A $\quad 17 \sim 23 \mathrm{~cm}$

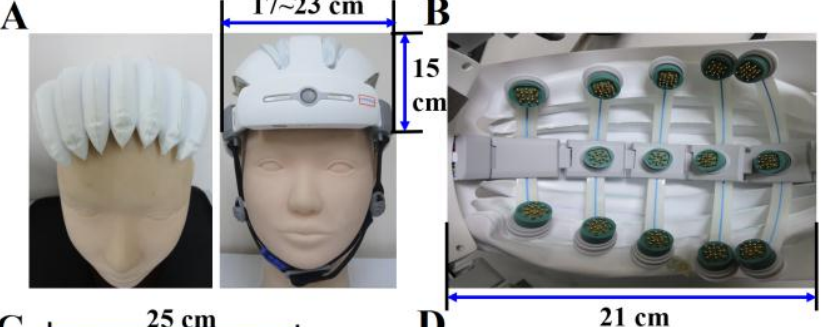

C

D

$21 \mathrm{~cm}$

Uninflated

Gasbag

Inflatable

Gasbag

2

3 


\section{$1 \quad$ Figure 5.}

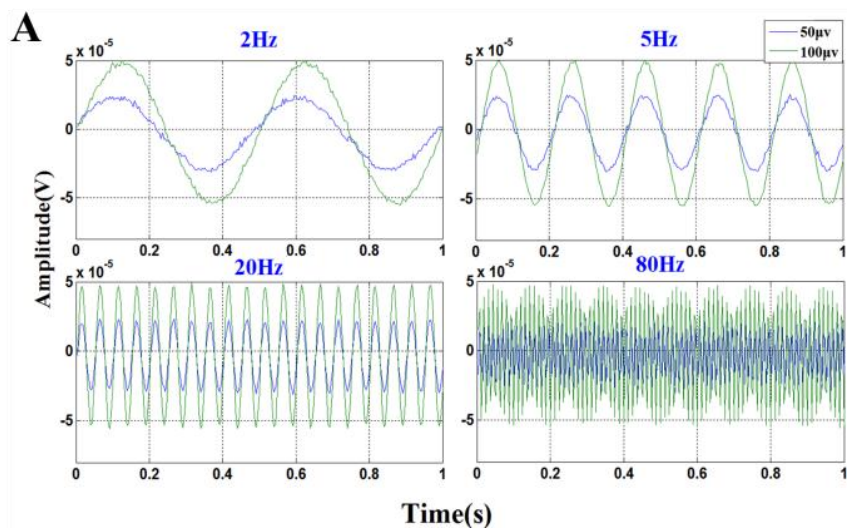

2

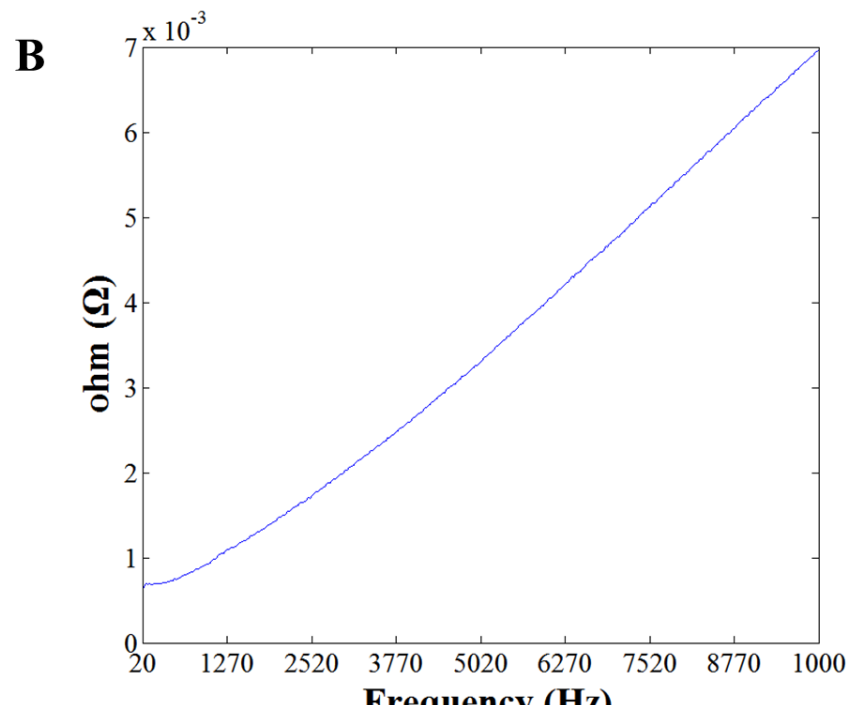


$1 \quad$ Figure 6.

Subject 1

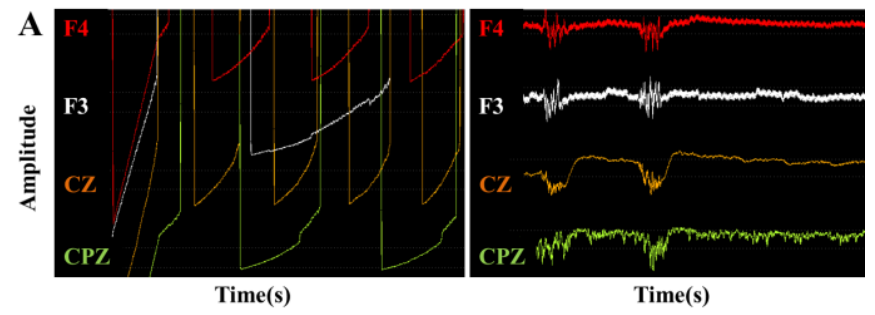

Subject 2

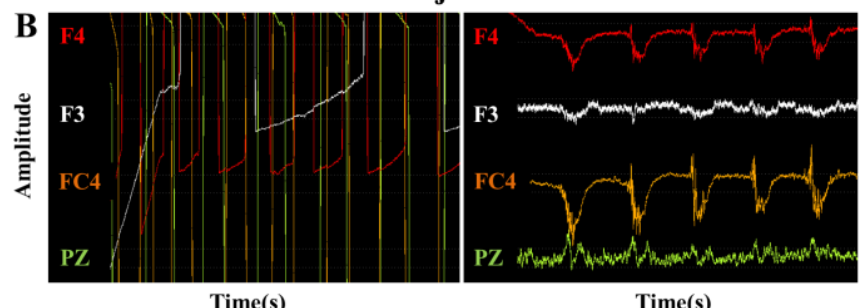

2

3

4 
$1 \quad$ Figure 7.
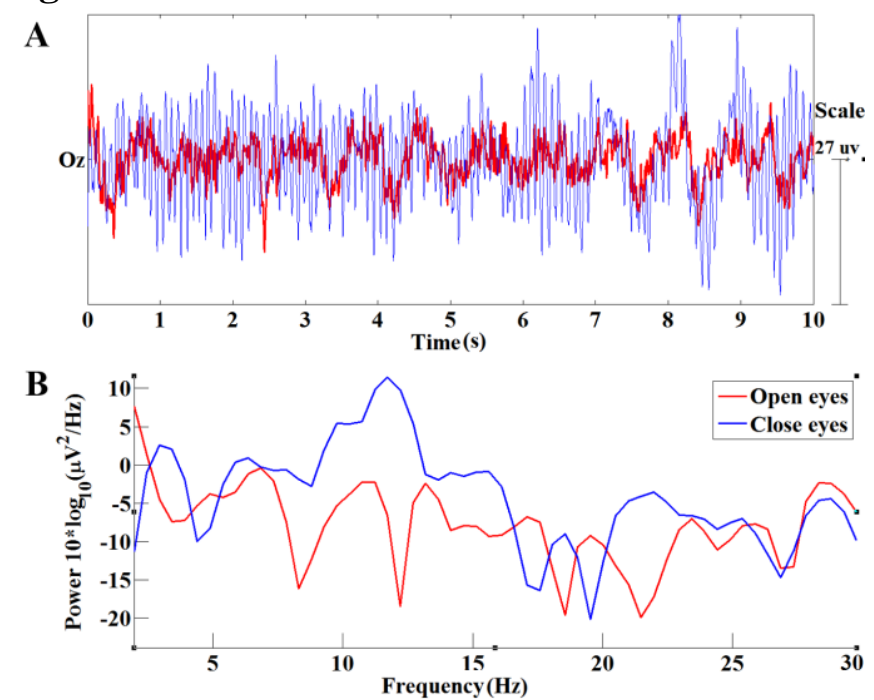
Figure 8.

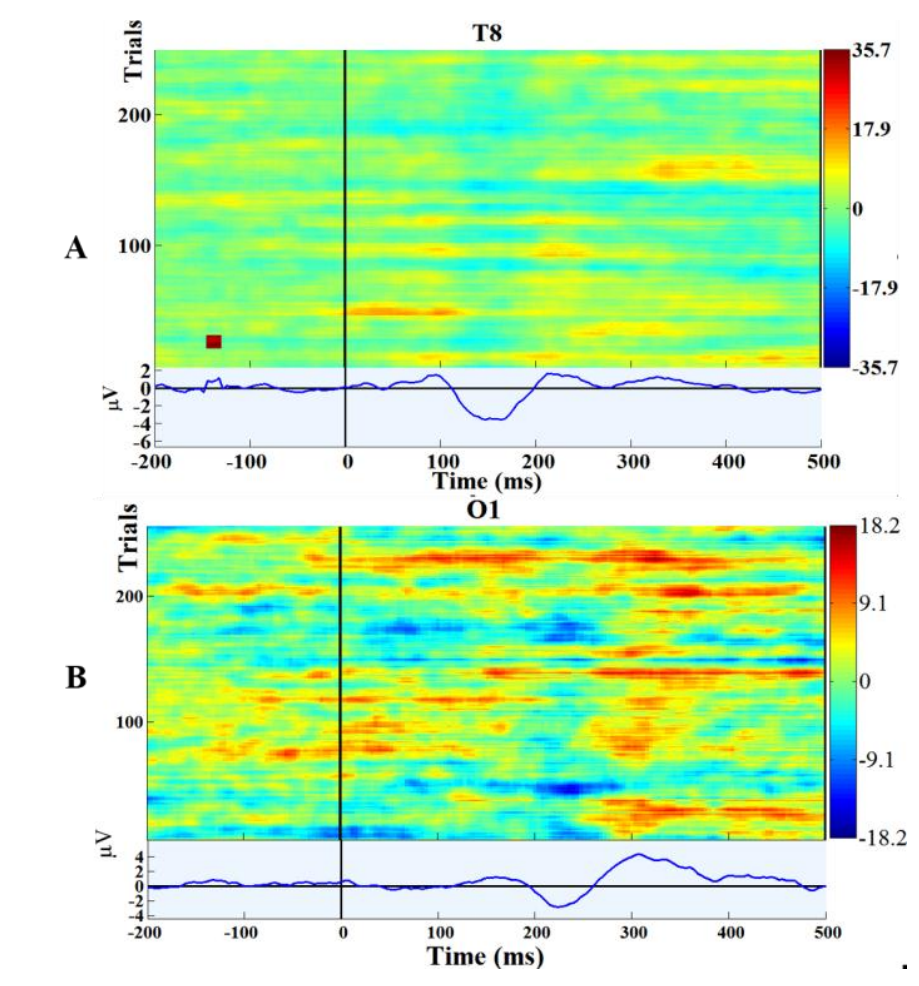

3 
$1 \quad$ Figure 9.

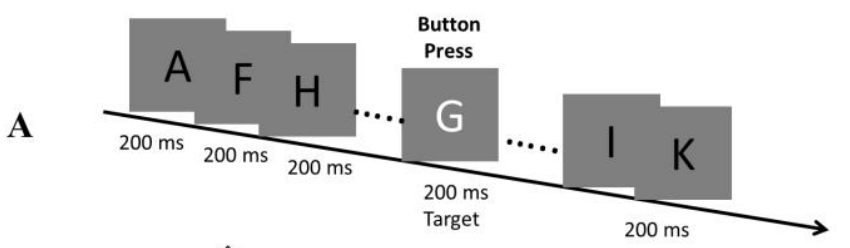

2
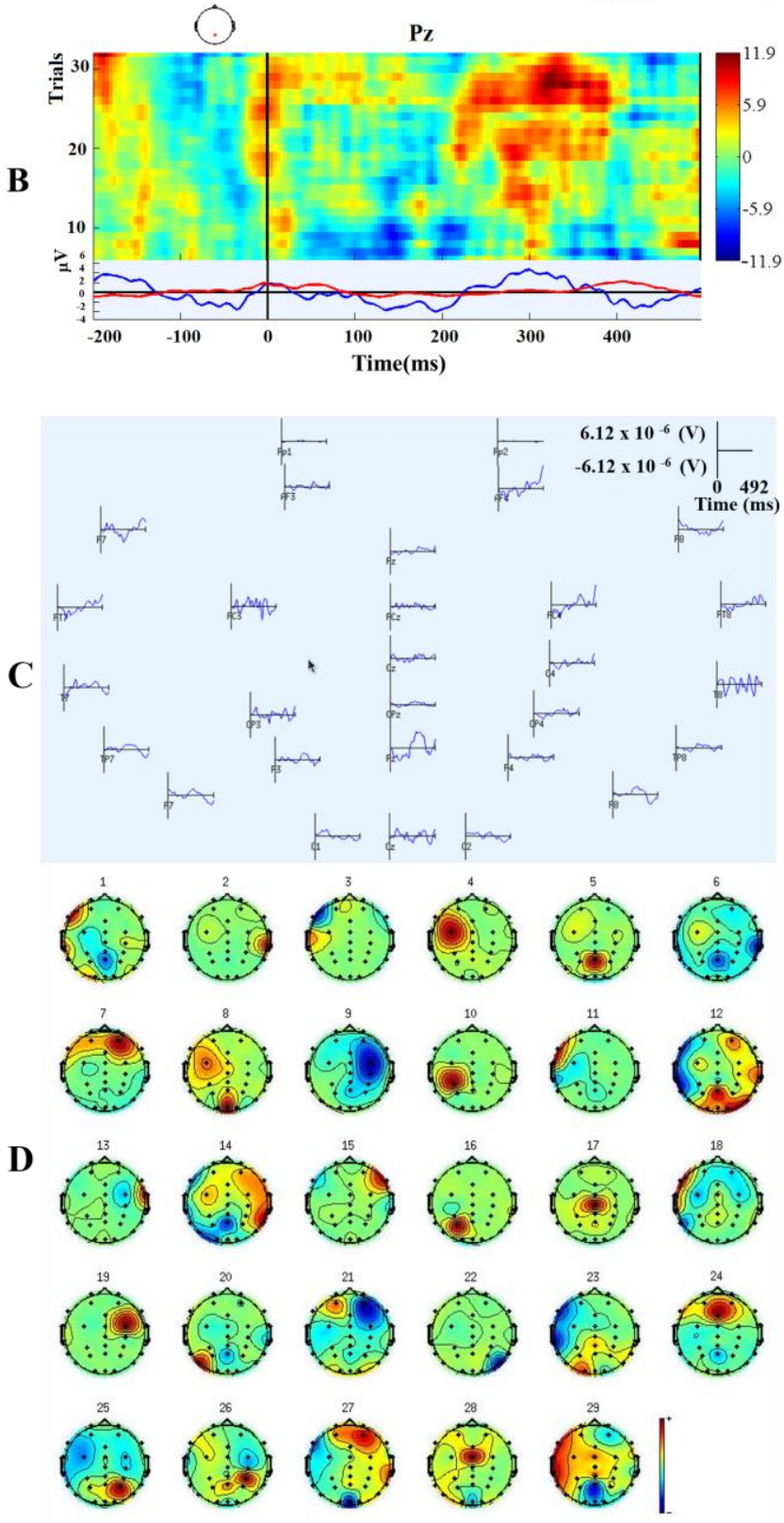